\title{
Variability of CHXR 20: accretion, extinction, spots or a companion?
}

\section{Taisiya G. Kopytova ${ }^{1,2}$ Viki Joergens ${ }^{1,3}$, Aurora Sicilia-Aguilar ${ }^{4}$, Maria V. Rodríguez-Ledesma ${ }^{1,5}$ and Reinhard Mundt ${ }^{1}$}

${ }^{1}$ Max-Planck Institut für Astronomie, Königstuhl 17, 69117 Heidelberg, Germany email: kopytova@mpia.de

\begin{abstract}
${ }^{2}$ International Max-Planck Research School for Astronomy and Cosmic Physics at the University of Heidelberg, IMPRS-HD, Germany ${ }^{3}$ Zentrum für Astronomie, Institut für Theoretische Astrophysik, Albert-Ueberle-Str. 2, 69120 Heidelberg, Germany ${ }^{4}$ Departamento de Física Teórica, Facultad de Ciencias, Universidad Autónoma de Madrid Cantoblanco, 28049 Madrid, Spain ${ }^{5}$ Institut für Astrophysik, Georg-August-Universität, Friedrich-Hund-Platz, 37077 Göttingen, Germany
\end{abstract}

\begin{abstract}
We study the photometric variability of a pre-main sequence star $\left(\mathrm{K} 6,0.9 \mathrm{M}_{\odot}\right)$ CHXR 20. We test several scenarios for the variability including variable accretion, variable extinction, cool and hot spots on the stellar surface and the presence of a potential companion.
\end{abstract}

Keywords. stars: activity, stars: pre-main sequence, stars: spots, stars: planetary systems: protoplanetary disks

\section{Introduction}

CHXR 20 (K6, Luhman 2004, 2007; $0.9 \mathrm{M}_{\odot}$, based on models by Siess et al. 2000) is a pre-main sequence member of the Chameleon I dark cloud. Its SED shows an infrared excess (Luhman et al. 2008) pointing to the presence of a circumstellar disk. Lawson \& Crause (2009) report a V-band variability of CHXR 20 suggesting that it is a candidate for an eclipsing binary system with an orbital period of 7.7 days.

In this work, we study the variability of CHXR 20 using available 2MASS (Cutri et al. 2003), WISE (Wright et al. 2010) and Spitzer (Luhman et al. 2008) data, and additionally, our own observations obtained with WFI and FEROS at the $2.2 \mathrm{~m}$ telescope in La Silla, Chile.

\section{Observations}

The $I_{c}$-band lightcurve obtained with WFI has a quasi-periodic character. The period search analysis determines a period of the variations of 7.0 days, which is consistent with the study of Lawson \& Crause (2009).

Available JHKs data from 2MASS point to the strong variability in the near-infrared, though the mid-infrared fluxes $(3.6,4.5,5.8,8.0,24.0 \mu \mathrm{m}$, Spitzer; 3.4, 4.6, 12, $22.0 \mu \mathrm{m}$, WISE) do not change significantly (Fig. 1).

High-resolution FEROS spectra show activity indicators: Ca II infrared lines and a highly variable $\mathrm{H} \alpha$ feature. The amplitude of radial velocity variations is $3.4 \mathrm{~km} \mathrm{~s}^{-1}$, though the available data (eight observational epochs) does not allow us to conclude if these variations are periodic or not. 


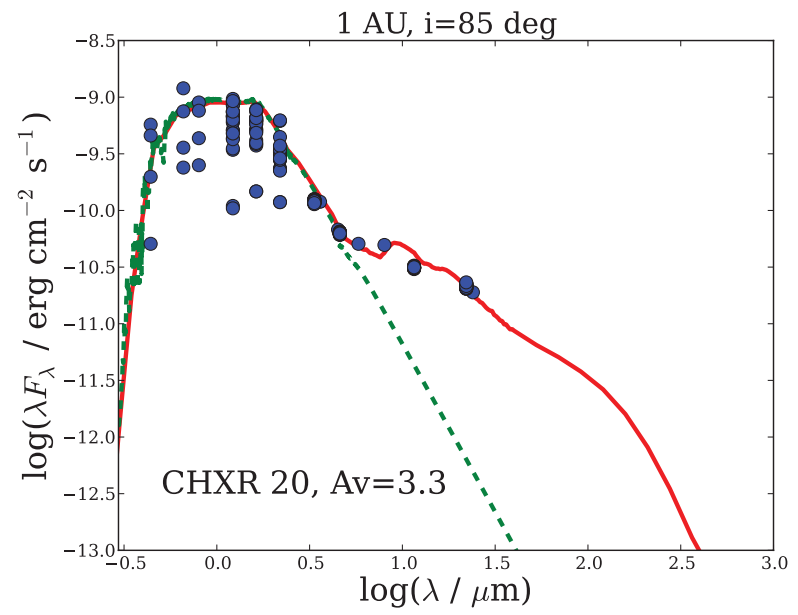

Figure 1. The spectral energy distribution of CHXR 20. The dashed line is a model photosphere. The disk modeling (solid line) with RADMC points to the presence of an inner gap in the disk.

\section{Discussion and conclusions}

Using the relation between color and magnitudes in the near-infrared, we notice that CHXR 20 gets redder when fainter. This allows us to exclude variable accretion from the potential sources of the photometric variability. On the other hand, the same colormagnitude relation is consistent with the reddening vector for CHXR 20 which was calculated following Cardelli et al. (1989). The latter favors the variable extinction origin of the near-infrared variability of CHXR 20.

Using the method proposed by Carpenter et al. (2001), we estimate that cool spots would not cause any photometric variability. However, hot spots with temperature 9000$10000 \mathrm{~K}$ covering $20-30 \%$ of the stellar surface could cause the variability in the optical but not in the near-infrared.

The SED modeling using RADMC (Dullemond \& Domonik 2004) suggests a possibility of the presence of an inner gap in the disk (Fig. 1). Given the radial velocity variations of $3.4 \mathrm{~km} \mathrm{~s}^{-1}$ and the period of photometric variability of 7 days, we tentatively suggest that the gap could be cleared-out by a companion with the mass of $15 \mathrm{M}_{J u p}$ at a maximum separation of $\sim 0.1 \mathrm{AU}$. The presence of the companion would also explain the observed photometric variability.

\section{References}

Cardelli, J. A., Clayton, G. C., \& Mathis, J. S. 1989, ApJ, 345, 245

Carpenter, J. M., Hillenbrand, L. A., \& Skrutskie, M. F. 2001, AJ, 121, 3160

Cutri, R. M., Skrutskie, M. F., van Dyk, S., Beichman, C. A., et al. 2000, VizieR Online Data Catalog

Dullemond, C. P. \& Dominik, C. 2004, A\&A, 417, 159

Lawson, W. A. \& Crause, L. A. 2009, PASA, 26, 31

Luhman, K. L. 2004, ApJ, 602, 816

Luhman, K. L. 2007, ApJS, 173, 104

Luhman, K. L., Allen, L. E., Allen, P. R., Gutermuth, R. A., Hartmann, L., Mamajek, E. E., Megeath, S. T., Myers, P. C., \& Fazio, G. G. 2008, ApJ, 675, 1375

Siess, L., Dufour, E., \& Forestini, M. 2000, A\& $A$, 358, 593

Wright, E. L., Eisenhardt, P. R. M., Mainzer, et al. 2010, AJ, 140, 1868 